\title{
Transition in Crystallization Rates of Fractionated Polyethylenes
}

\author{
Shuichi SAWADA and Takuhei NosE \\ Department of Polymer Chemistry, Tokyo Institute of Technology, \\ Ookayama, Meguro-ku, Tokyo 152, Japan.
}

(Received May 22, 1978)

\begin{abstract}
The kinetics of fractionated polyethylenes was investigated by the method of dilatometry. A discontinuous change in crystallization rates was observed for molecular weights 4300 and 17300 . This discontinuity is shown to be an important factor in clarifying the mechanism of the formation of extended-chain crystals. Extended-chain crystals were obtained within the region where folded-chain crystals were prohibited. Extended-chain crystallization had a mode of three-dimensional growth. It is suggested that the formation of extended-chain crystals at atmospheric pressure is distinct from that under high pressure. A theory of polymer crystallization is proposed in order to explain the transition in crystallization rates.
\end{abstract}

KEY WORDS Polyethylene / Crystallization Rates / Extended-Chain Crystals / Avrami Exponent / Stochastic Process / Surface Nucleation Rate /

Extended-chain crystals can be produced at atmospheric pressure, depending on the crystallization conditions. ${ }^{1}$ It was shown, however, that it became harder to give rise to extendedchain crystals as the molecular weight of polymers was increased. In extended-chain crystallization under high pressure, the situation is completely reversed, that is, extended-chain crystals are easily produced with increasing molecular weight. $^{2-4}$

As regards this inconsistency, we consider that it is necessary to clear up whether the mechanism of the formation of extended-chain crystals at atmospheric pressure and at high pressures is the same or not. Under high pressure, extended and folded-chain crystallization are considered to be rival processes from the kinetical point of view. Meanwhile, little is known about the mechanism of the formation of extended-chain crystals at atmospheric pressure, because the kinetics of extended-chain crystals has not been fully investigated.

In this study the crystallization rates of three kinds of fractionated linear polyethylenes with different molecular weights were measured in order to get information about the mechanism of the formation of extended-chain crystals at atmo- spheric pressure, and the growth mode of extendedchain crystallization was compared with that at high pressures.

We also present a theory of the growth rates of $m$-times folded-chain crystals with finite molecular weight for the purpose of explaining the transition in crystallization rates observed in this experiment.

\section{EXPERIMENTAL}

The materials used in this work were the viscosity-average molecular weights $M_{v}=4300,17300$. and 150000 of linear polyethylene fractions $\left(M_{w} / M_{n}\right.$ was in the range 1.2-1.3) that were obtained from Sholex $6000 \mathrm{C}$ by the column fractionation method.

We studied crystallization kinetics by using dilatometric techniques. The materials which were originally in the form of powder were melted and cooled to room temperature under vacuum. About $300 \mathrm{mg}$ of the materials were put into the dilatometer, whose diameter was $0.8 \mathrm{~mm}$; after being filled with fresh mercury, the dilatometer was evacuated overnight. The dilatometer was placed in a silicone oil bath in which the temperature was kept at about $160^{\circ} \mathrm{C}$ in order to assure the complete melting of the sample. After holding 


\section{S. SAWAdA and T. NosE}

the dilatometer in that bath for about $60 \mathrm{~min}$, it was quickly transferred to another silicone oil bath whose temperature was set at a predetermined crystallization temperature and controlled to better than $0.03^{\circ} \mathrm{C}$. Although it took about $2-3 \mathrm{~min}$ to reach temperature equilibrium, the time of transfer was taken as the starting time for crystallization kinetics. The height of the mercury in the dilatometer was measured as a function of time.

Experimental data were analyzed by using the Avrami equation

$$
X=\exp \left(-k t^{n}\right)
$$

where $1-X$ is the relative degree of completion of the process at time $t, k$ is a crystallization rate constant, and $n$ is the Avrami exponent which characterizes the growth mode of crystallization. $X$ was evaluated by the relation

$$
X=\frac{h_{\mathrm{t}}-h_{\infty}}{h_{0}-h_{\infty}}
$$

where $h_{0}$ is the mercury height of the dilatometer at initial time, $h_{\mathrm{t}}$ at time $t$, and $h_{\infty}$ the final level of the mercury column. The crystallization rate constant $k$ was estimated from $\tau_{0.1}$, the time required for the $10 \%$ of the transformation to occur.

$$
\log k=-n \log \tau_{0.1}+\log \left(\ln \frac{10}{9}\right)
$$

Surface replicas of the materials fractured in liquid nitrogen were investigated by using an electron microscope. The measurements of the fold period of the materials which crystallized isothermally were carried out with the method of small-angle $\mathrm{X}$-ray scattering, radiating each sample for about $20 \mathrm{~h}$.

\section{RESULTS AND DISCUSSION}

\section{Transition in Crystallization Rates}

The crystallization rate constant $k$ is plotted against $T_{\mathrm{m}}{ }^{0} / T_{\mathrm{c}} \Delta T$ for three samples with different molecular weights in Figure 1, where $T_{\mathrm{m}}{ }^{0}$ denotes the equilibrium temperature, $T_{\mathrm{c}}$ the crystallization temperature, and $\Delta T$ the degree of supercooling. A linear relation was obtained for the sample with the highest molecular weight $\left(M_{v}=150000\right)$. However, two straight lines with different slopes were required to fit the data for the other samples

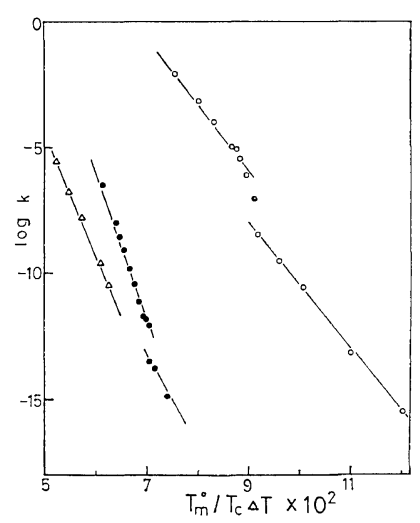

Figure 1. Plot of $\log k$ against $T_{\mathrm{m}}{ }^{0} / T_{\mathrm{c}} \Delta T$ for molecular weights: $\bigcirc, 4300 ; \bullet, 17300 ; \triangle, 150000$.

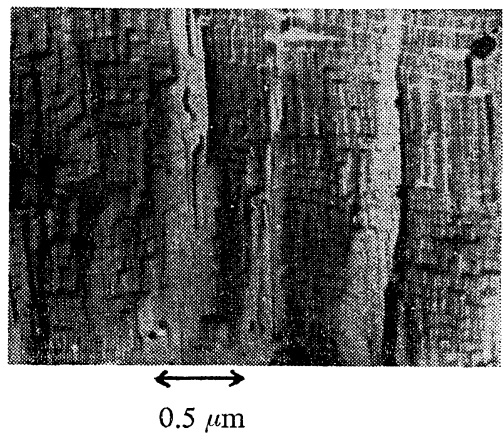

Figure 2. Electron micrograph of a fracture surface of the materials crystallized at $126.0^{\circ} \mathrm{C}$ for molecular weight of 4300 .

with lower molecular weights $\left(M_{v}=4300\right.$ and 17300). For molecular weight 150000 , only folded-chain crystals are assumed to be produced under the usual experimental conditions; therefore, crystallization rates were expressed by a single line. In the case of molecular weights 4300 and 17300 , which give two straight lines, the crystallization temperature can be divided into two regions at the turning point of the crystallization rates: a high crystallization temperature region and a low one.

For molecular weight 4300 , the crystals obtained in the high crystallization temperature region were shown to have a fully extended-chain structure (average lamellar thickness of $400 \AA$ ) from the electron microscopic studies. This is shown in Figure 2. On the other hand, the crystals produced in 


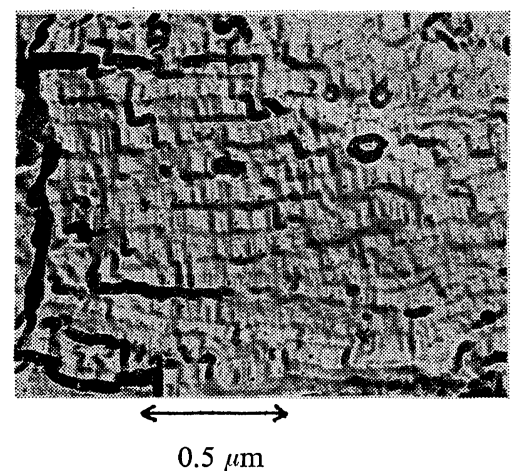

Figure 3. Electron micrograph of a fracture surface of the materials crystallized at $127.4^{\circ} \mathrm{C}$ for molecular weight 17300 .

the low crystallization temperature region had a lamellar thickness of about $200 \AA$ from the studies of small-angle X-ray scattering. This corresponds to once folded-chain crystals.

The transition in crystallization rates was supposed to be caused by the change of structure from once folded-chain to extended-chain crystals. Therefore, it is considered that extended-chain crystallization took place in the region where folded-chain crystallization was prohibited. It is thus not the case: under high pressure extended-chain and folded-chain crystallization are rival processes.

In the case of molecular weight 17300 , the high crystallization temperature region produced the crystals with the lamelar thickness of about $800 \AA$, which correspond to once folded-chain crystals; this is shown in Figure 3. It is suggested that the discontinuous change was caused by the change of structure from almost twice folded-chain to once folded-chain crystals. No crystals with the contour length of a molecule (about $1560 \AA$ ) were produced in this work. Since it takes a long time, it must be very difficult to produce extendedchain crystals.

\section{Avrami Exponent}

The double logarithm of $X$ was plotted against the logarithm of time $t$. The representative data are shown in Figures 4-6. In the straight line portions, the Avrami exponent $n$ was estimated by the method of least squares, and is tabulated in Table I.

For molecular weight 150000 , the exponent $n$

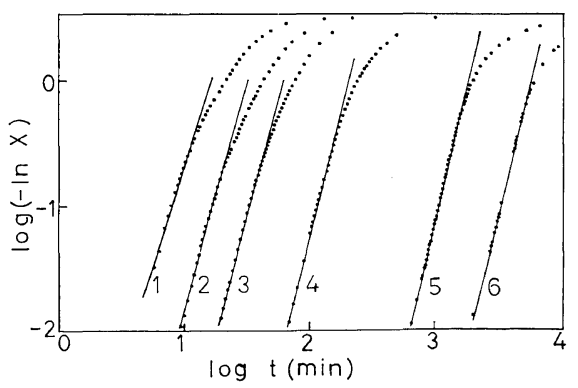

Figure 4. Double logarithmic plot of $X$ at (1) 122.9, (2) 123.6, , (3) 124.0 , (4) 124.6, , (5) 126.0 , and (6) $126.8^{\circ} \mathrm{C}$ for molecular weight of 4300 .

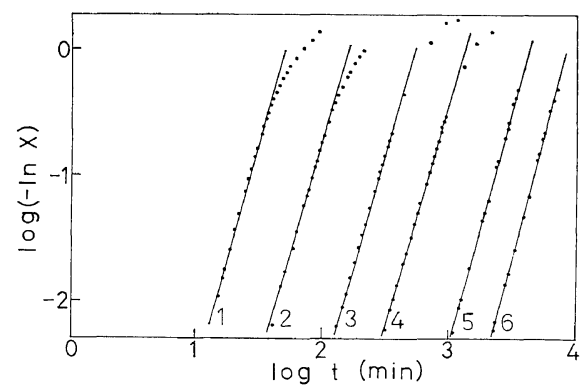

Figure 5. Double logarithmic plot of $X$ at (1) 125.2, (2) 125.9, , (3) 126.4 , (4) 126.9, (5) 127.6 , and (6) $128.1^{\circ} \mathrm{C}$ for molecular weight of 17300 .

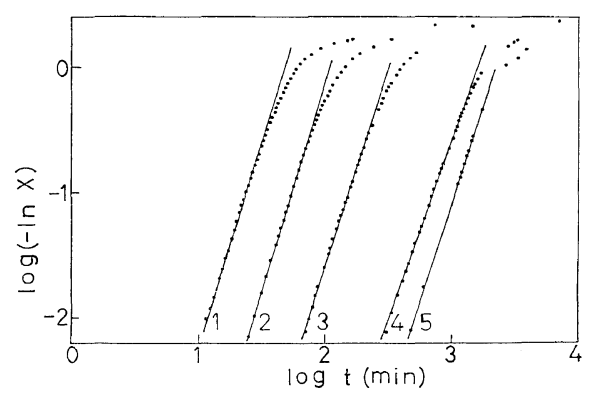

Figure 6. Double logarithmic plot of $X$ at (1) 126.0, (2) 127.0 , (3) 128.0 , (4) 129.1 , and (5) $129.5^{\circ} \mathrm{C}$ for molecular weight of 150000 .

was almost independent of the crystallization temperatures studied at a valve of about three, while $n$ was dependent on the crystallization conditions for molecular weights 4300 and 17300 . Above all, in the case of molecular weight 4300 , $n$ varied from 3 to 4 . The value of three was typical of the crystals at the lower crystallization tem- 
Table I. Avrami exponent $n$

\begin{tabular}{cccccc}
\multicolumn{2}{c}{$M_{v}=4300$} & \multicolumn{2}{c}{$M_{v}=17300$} & \multicolumn{2}{c}{$M_{v}=150000$} \\
$T_{\mathrm{c}},{ }^{\circ} \mathrm{C}$ & $n$ & $T_{\mathrm{c}},{ }^{\circ} \mathrm{C}$ & $n$ & $T_{\mathrm{c}},{ }^{\circ} \mathrm{C}$ & $n$ \\
\hline 121.6 & 2.8 & 125.2 & 3.2 & 126.0 & 3.3 \\
122.4 & 3.1 & 125.8 & 3.3 & 127.0 & 3.3 \\
122.9 & 3.2 & 125.9 & 3.2 & 128.0 & 3.1 \\
123.4 & 3.3 & 126.2 & 3.3 & 129.1 & 2.9 \\
123.5 & 3.3 & 126.4 & 3.2 & 129.5 & 3.1 \\
123.6 & 3.5 & 126.7 & 3.2 & & \\
123.8 & 3.6 & 126.9 & 3.2 & & \\
124.0 & 3.9 & 127.1 & 3.1 & & \\
124.1 & 4.2 & 127.2 & 3.2 & & \\
124.6 & 4.1 & 127.3 & 3.3 & & \\
125.1 & 3.9 & 127.4 & 3.7 & & \\
126.0 & 4.0 & 127.6 & 3.5 & & \\
126.8 & 4.1 & 128.1 & 3.6 & & \\
& & & &
\end{tabular}

peratures, and higher crystallization temperatures gave rise to the crystals with the value of four.

As the crystals obtained in the high crystallization temperature region were extended-chain crystals, we assume that extended-chain crystallization at atmospheric pressure has a mode of three-dimensional growth. This is distinct from the mode of growth of extended-chain crystals at high pressures, which is expressed as a one-dimensional growth mode. ${ }^{5}$ We consider that this difference in the mode of growth reflects the distinction of the mechanism of the growth between extended-chain crystallization at atmospheric pressure and high pressures.

\section{Theoretical Consideration}

In connection with the transition in crystallization rates we developed the theory of the crystal growth of $m$-times folded-chain crystals for finite molecular weight. Growth rates $G(\mathrm{~cm} / \mathrm{s})$ and surface nucleation rate $J$ (nuclei/s site) were correlated by the following relation.

$$
G=c J N_{0}
$$

where $c$ is a constant and $N_{0}$ the average number of nucleation sites. This relation will hold in the case of nucleation-controlled crystal growth where the surface nucleation rate is much smaller than the lateral growth rate to complete the growth strip to form a monolayer surface. ${ }^{6-8}$

Now, we estimate the surface nucleation rate $J$ by using the method of stochastic processes, which had been used to describe the nucleation rates of folded-chain crystals. $^{6,7,9}$ But these methods have not been applied to the case of the crystal growth of a few times folded-chain crystals.

For the sake of simplifying this treatment we made the following assumptions for the formation of folded-chain crystals. Firstly the polymer is a monodisperse one. Secondly, the chain ends never enter into the interior of the crystals and a molecule folds integral times.

We denote $\alpha_{\nu-1}$ and $\beta_{\nu}$ as the forward and backward transition rates between the states $\nu-1$ and $\nu$, respectively. The net transition rate $J_{\nu}$ between the states $\nu-1$ and $\nu$ is

$$
J_{\nu}=\alpha_{\nu-1} N_{\nu-1}-\beta_{\nu} N_{\nu}
$$

where $N_{\nu-1}$ and $N_{\nu}$ are the occupation numbers at the states $\nu-1$ and $\nu$, respectively. The occupation number $N_{\nu}$ is time dependent and given by

$$
\frac{\mathrm{d} N_{\nu}}{\mathrm{d} t}=J_{\nu}-J_{\nu+1}
$$

In the case of finite molecular weight, after a molecule is incorporated to complete $m$ stems on the crystal substrate, a different molecule must be incorporated to complete another $m$ stems. This process will be repeated over and over again, so that transition rates have a periodicity.

We denote the transition rates in the following way. In the first sequence of $m$ stems, the forward and backward transition rates between the states $\nu=0$ and $\nu=1$ are $\alpha_{0}$ and $\beta_{1}$, respectively. The subsequent transition rate pairs are the same, and can be expressed as $\alpha_{1}$ and $\beta_{2}$. In the second sequence of $m$ stems, only the first transition rate pairs are distinct from the case of the first sequence and are given by $\alpha_{1}$ and $\beta_{2}$. The transition rates for the following sequence of $m$ stems are the same as those for the second sequence of $m$ stems.

For the first sequence, the net transition rates are

$$
\begin{gathered}
J_{1}=\alpha_{0} N_{0}-\beta_{1} N_{1} \\
J_{2}=\alpha_{1} N_{1}-\beta_{2} N_{2} \\
\cdots \cdots \\
J_{m}=\alpha_{1} N_{m-1}-\beta_{2} N_{m}
\end{gathered}
$$

For the subsequent sequence, the net transition rates for $\nu=2,3,4, \ldots$ are 


$$
\begin{aligned}
J_{(\nu-1) m+1}= & \alpha_{2} N_{(\nu-1) m}-\beta_{2} N_{(\nu-1) m+1} \\
J_{(\nu-1) m+2}= & \alpha_{1} N_{(\nu-1) m+1}-\beta_{2} N_{(\nu-1) m+2} \\
& \cdots \cdots \cdots \\
J_{\nu m}= & \alpha_{1} N_{(\nu-1) m+(m-1)}-\beta_{2} N_{\nu m}
\end{aligned}
$$

Steady-state conditions were imposed. Therefore, the net transition rates are all equal and can be described by $J$. Each equation in eq 7 except the first is multiplied by $\left(\beta_{1} / \alpha_{1}\right)\left(\beta_{2} / \alpha_{1}\right)^{i-2}, i=2,3$, $\cdots, m$, and all equations are added up to obtain

$$
J=A_{0} N_{0}-B_{1} N_{m}
$$

where

$$
\begin{aligned}
& A_{0}=\frac{\alpha_{0}}{1+\left(\beta_{1} / \alpha_{1}\right) \sum_{k=1}^{m-1}\left(\beta_{2} / \alpha_{1}\right)^{k-1}} \\
& B_{1}=\frac{\beta_{1}\left(\beta_{2} / \alpha_{1}\right)^{m-1}}{1+\left(\beta_{1} / \alpha_{1}\right) \sum_{k=1}^{m-1}\left(\beta_{2} / \alpha_{1}\right)^{k-1}}
\end{aligned}
$$

Each equation in eq 8 for $\nu=2,3,4, \cdots$ is multiplied by $\left(\beta_{2} / \alpha_{1}\right)^{i-1}, i=2,3, \cdots, m$, except the first, and all equations are added up to obtain

$$
J=A_{1} N_{(\nu-1) m}-B_{2} N_{\nu m} \quad \nu=2,3,4, \cdots
$$

where

$$
\begin{aligned}
& A_{1}=\frac{\alpha_{2}}{\sum_{k=1}^{m}\left(\beta_{2} / \alpha_{1}\right)^{k-1}} \\
& B_{2}=\frac{\beta_{2}\left(\beta_{2} / \alpha_{1}\right)^{m-1}}{\sum_{k=1}^{m}\left(\beta_{2} / \alpha_{1}\right)^{k-1}}
\end{aligned}
$$

Each equation in eq 12 is multiplied by $\left(B_{1} / A_{1}\right)$ $\left(B_{2} / A_{1}\right)^{\nu-2} \nu=2,3,4, \cdots$ and the resulting equations and eq 9 are added up to obtain

$$
\begin{gathered}
J=\frac{A_{0} N_{0}}{1+\left(B_{1} / A_{1}\right) \sum_{k=1}^{\nu-1}\left(B_{2} / A_{1}\right)^{k-1}} \\
-\frac{B_{1}\left(B_{2} / A_{1}\right)^{\nu-1} N_{\nu m}}{1+\left(B_{1} / A_{1}\right) \sum_{k=1}^{\nu-1}\left(B_{2} / A_{1}\right)^{k-1}}
\end{gathered}
$$

The condition $B_{2} / A_{1}<1$ has to be satisfied in order to assure nucleation growth, and if $\nu$ is large we obtain the steady-state solution

$$
J=\frac{A_{0}\left(A_{1}-B_{2}\right) N_{0}}{A_{1}+B_{1}-B_{2}}
$$

$J$ is determined by choosing appropriate transition rates through eq $10,11,13$, and 14 . The transition rates were chosen in the following way.

$$
\begin{aligned}
& \alpha_{0}=f \exp \left\{-\left(a b \sigma_{\mathrm{ee}}+2 b l \sigma-0.5 a b l \Delta g\right) / k T\right\} \\
& \alpha_{1}=f \exp \left\{\left(a b \sigma_{\mathrm{ee}}+2 a b \sigma_{\mathrm{ef}}-0.5 a b l \Delta g\right) / k T\right\} \\
& \alpha_{2}=f \exp \left\{-\left(a b \sigma_{\mathrm{ee}}-0.5 a b l \Delta g\right) / k T\right\} \\
& \beta_{1}=\beta_{2}=f \exp (-0.5 a b l \Delta g / k T)
\end{aligned}
$$

where $\sigma_{\mathrm{ee}}, \sigma_{\mathrm{ef}}$, and $\sigma$ are the excess free energies of the end group, fold surface, and lateral surface, respectively, $l=L / m$ ( $L$ is the contour length of a molecule), the stem length which corresponds to the lamellar thickness, $a$ the molecular width, $b$ the layer thickness, and $\Delta g$ the bulk free energy of fusion. $f$ expresses the transport term at the crystal-liquid interface. In the four transition rates, $\alpha_{0}$ shows the transition rate on the occasion of attaching the first stem of a molecule to the crystal substrate and $\beta_{1}$ is the transition rate of the detaching process. $\quad \alpha_{1}$ is the transition rate which is connected with creating a fold surface. $\alpha_{2}$ expresses the transition rate on the occasion of attaching the first stem of another molecule to the already deposited molecule.

The surface nucleation rate of extended-chain crystals is also obtained in a similar way. In this case, the forward transition rate $\alpha_{0 \mathrm{e}}$ for the first sequence is distinct from the forward transition rate $\alpha_{1 \mathrm{e}}$ in the following sequence and the backward transition rates $\beta_{1 \mathrm{e}}$ are assumed to be the same. The surface nucleation rate for extended-chain crystals is easily obtained,

$$
J=\alpha_{0 \mathrm{e}}\left(1-\frac{\beta_{1 \mathrm{e}}}{\alpha_{1 \mathrm{e}}}\right) N_{0}
$$

where

$$
\begin{aligned}
& \alpha_{0 \mathrm{e}}=f \exp \left\{-\left(2 a b \sigma_{\mathrm{ee}}+2 b L \sigma-0.5 a b L \Delta g\right) / k T\right\} \\
& \alpha_{1 \mathrm{e}}=f \exp \left\{-\left(2 a b \sigma_{\mathrm{ee}}-0.5 a b L \Delta g\right) / k T\right\} \\
& \beta_{1 \mathrm{e}}=f \exp (-0.5 a b L \Delta g / k T)
\end{aligned}
$$

By the combination of eq 4, 16, and 21, growth rates can be calculated and these are shown in Figures 7 and 8.

In eq $16, B_{2} / A_{1}=1$ means that crystals are in an equilibrium state with the surrounding melt. Therefore, $(m-1)$ times folded-chain crystals have a limit of crystallization temperatures at which further crystal growth stops. This limiting point 


\section{S. SAWADA and T. NoSE}

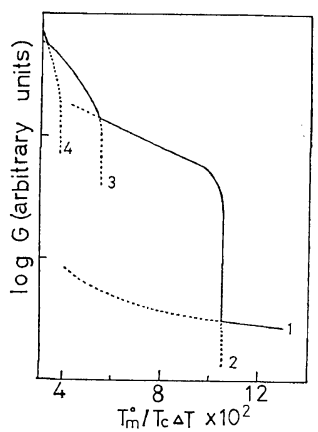

Figure 7. Plot of growth rate $G$ against $T_{\mathrm{m}}{ }^{0} / T_{\mathrm{c}} \Delta T$ for lamellar thickness (1) $l=L$, (2) $l=L / 2$, (3) $l=L / 3$, and (4) $l=L / 4$. Calculated for $T_{\mathrm{m}}{ }^{0}=409 \mathrm{~K}, L=3.9$ $\times 10^{-6} \mathrm{~cm}$ (corresponds to molecular weight 4300 ), $\sigma=7 \mathrm{erg} / \mathrm{cm}^{2}, \sigma_{\mathrm{ee}}=10 \mathrm{erg} / \mathrm{cm}^{2}$, and $\sigma_{\text {ef }}=120 \mathrm{rg} / \mathrm{cm}^{2}$.

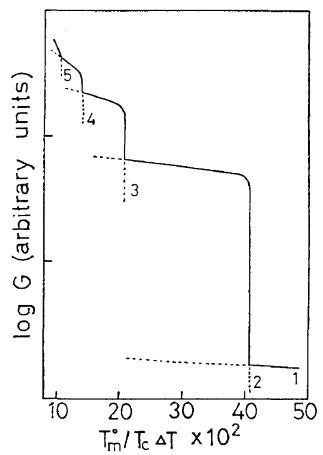

Figure 8. Plot of growth rate $G$ against $T_{\mathrm{m}} 0 / T_{\mathrm{c}} \Delta T$ for lamellar thickness (1) $l=L$, (2) $l=L / 2$, (3) $l=L / 3$, (4) $l=L / 4$, and (5) $l=L / 5$. Calculated for $T_{\mathrm{m}}{ }^{0}=$ $416 \mathrm{~K}, L=1.56 \times 10^{-5} \mathrm{~cm}$ (corresponds to molecular weight 17300), $\sigma=7 \mathrm{erg} / \mathrm{cm}^{2}, \sigma_{\mathrm{e \theta}}=10 \mathrm{erg} / \mathrm{cm}^{2}$, and $\sigma_{\text {ef }}=120 \mathrm{erg} / \mathrm{cm}^{2}$.

is derived by using eq 13 and 14 :

$$
T_{\mathrm{c}}=\left[1-\frac{m \sigma_{\mathrm{ee}}+2(m-1) \sigma_{\mathrm{ef}}}{L \Delta h}\right] T_{\mathrm{m}}{ }^{0}
$$

where $\Delta h$ is the heat of fusion. If $m=2$, the clystallization temperature above which foldedchain crystals do not grow can be obtained

$$
T_{\mathrm{c}}=\left[1-\frac{2 \sigma_{\mathrm{ee}}+2 \sigma_{\mathrm{ef}}}{L \Delta h}\right] T_{\mathrm{m}}{ }^{0}
$$

At this temperature, growth rates show the discontinuous change from once folded-chain to extended-chain crystallization. This transition becomes sharper as the molecular weight is in- creased; moreover, growth rates show an abrupt change from twice folded-chain to once foldedchain crystallization for higher molecular weights, as is shown in Figures 7 and 8.

Although for simplification only the contribution of the growth rates in the crystallization process was taken into account in this model, this may be justified from the fact that the growth rates reflect the overall crystallization rates because of the similarity of the dependence of both rates on crystallization temperature. The attempt described in this paper could give a qualitative explanation of the transition in crystallization rates for the fractionated polyethylenes studied. We assume that a polymer with lamellar thickness which varied in a stepwise way, such as poly(ethylene oxide), satisfies this model reasonably well. It was reported that the crystal growth rate of poly(ethyleneoxide) showed the clear dependence on the number of folds. ${ }^{10}$

In conclusion we suggest that the mechanism of the formation of extended-chain crystallization at atmospheric pressure is different from that at high pressures. It is considered that the formation of extended-chain crystals for high molecular weight at atmospheric pressure is difficult, mostly because of the high free energy in nucleation events due to the large lamellar thickness.

\section{REFERENCES}

1. F. R. Anderson, J. Appl. Phys., 35, 64 (1964).

2. K. Kato, T. Nose, and T. Hata, Rep. Prog. Polym. Phys. Jpn., 14, 187 (1971).

3. T. Hatakeyama, T. Hashimoto, T. Ishida, M. Ohkuma, M. Kyotani, and H. Kanetsuna, Rep. Prog. Polym. Phys. Jpn., 14, 199 (1971).

4. D. C. Bassett and B. Turner, Phill. Mag., 29, 285 (1974).

5. M. Kyotani and H. Kanetsuna, J. Polym. Sci., Part. A-2, 12, 2331 (1974).

6. I. C. Sanchez and E. A. Dimarzio, J. Chem. Phys., 55, 893 (1971).

7. J. I. Lauritzen and J. D. Hoffman, J. Appl. Phys., 44, 4340 (1973).

8. P. D. Calvert and D. R. Uhlmann, J. Appl. Phys., 43, 944 (1972).

9. F. C. Frank and M. Tosi, Proc. R. Soc., London Ser. A, 263, 323 (1961).

10. A. J. Kovacs and A. Gonthier, Kolloid-Z. Z Polym., 250, 530 (1972). 